\title{
Corneal Epithelial Findings in Patients with Multiple Myeloma Treated with Antibody-Drug Conjugate Belantamab Mafodotin in the Pivotal, Randomized, DREAMM-2 Study
}

Asim V. Farooq · Simona Degli Esposti · Rakesh Popat - Praneetha Thulasi · Sagar Lonial · Ajay K. Nooka · Andrzej Jakubowiak · Douglas Sborov · Brian E. Zaugg · Ashraf Z. Badros · Bennie H. Jeng $\cdot$ Natalie S. Callander $\cdot$ Joanna Opalinska $\cdot$ January Baron $\cdot$ Trisha Piontek Julie Byrne · Ira Gupta $\cdot$ Kathryn Colby Received: April 21, 2020 / Published online: July 25, 2020 (C) The Author(s) 2020, corrected publication 2020

\section{ABSTRACT}

Introduction: Patients with relapsed or refractory multiple myeloma (RRMM) represent an unmet clinical need. Belantamab mafodotin (belamaf; GSK2857916) is a first-in-class antibody-drug conjugate (ADC; or immunoconjugate) that delivers a cytotoxic payload, monomethyl auristatin F (MMAF), to myeloma cells. In the phase II DREAMM-2 study (NCT03525678), single-agent belamaf $(2.5 \mathrm{mg} / \mathrm{kg})$

A. V. Farooq $(\bowtie) \cdot$ A. Jakubowiak $\cdot$ K. Colby University of Chicago Medical Center, Chicago, IL, USA

e-mail: afarooq@bsd.uchicago.edu

S. Degli Esposti

NIHR Biomedical Research Centre at Moorfields Eye Hospital NHS Foundation Trust, UCL Institute of Ophthalmology, London, UK

R. Popat

University College London Hospitals, NHS

Foundation Trust, London, UK

P. Thulasi

Emory Eye Center, Emory University, Atlanta, GA, USA

S. Lonial · A. K. Nooka

Emory University, Winship Cancer Institute,

Atlanta, GA, USA

D. Sborov

Huntsman Cancer Institute, University of Utah, Salt Lake City, UT, USA demonstrated clinically meaningful anti-myeloma activity (overall response rate $32 \%$ ) in patients with heavily pretreated disease. Microcyst-like epithelial changes (MECs) were common, consistent with reports from other MMAFcontaining ADCs.

Methods: Corneal examination findings from patients in DREAMM-2 were reviewed, and the clinical descriptions and accompanying images (slit lamp microscopy and in vivo confocal microscopy [IVCM]) of representative events were selected. A literature review on corneal

\section{B. E. Zaugg}

Moran Eye Center, University of Utah, Salt Lake City, UT, USA

\section{A. Z. Badros}

University of Maryland School of Medicine, Baltimore, MD, USA

B. H. Jeng

Department of Ophthalmology and Visual Sciences, University of Maryland School of Medicine, Baltimore, MD, USA

\section{N. S. Callander}

University of Wisconsin, Carbone Cancer Center, Madison, WI, USA

J. Opalinska · J. Baron · T. Piontek · J. Byrne ·

I. Gupta

GlaxoSmithKline, Upper Providence, PA, USA 
events reported with other ADCs was performed.

Results: In most patients receiving single-agent belamaf (72\%; 68/95), MECs were observed by slit lamp microscopy early in treatment (69\% had their first event by dose 4). However, IVCM revealed hyperreflective material. Blurred vision (25\%) and dry eye (15\%) were commonly reported symptoms. Management of MECs included dose delays (47\%)/reductions (25\%), with few patients discontinuing due to MECs (1\%). The first event resolved in most patients (grade $\geq 2$ MECs and visual acuity [each $77 \%$ ], blurred vision [67\%], and dry eye [86\%]), with no reports of permanent vision loss to date. A literature review confirmed that similar MECs were reported with other ADCs; however, event management strategies varied. The pathophysiology of MECs is unclear, though the ADC cytotoxic payload may contribute to on- or offtarget effects on corneal epithelial cells.

Conclusion: Single-agent belamaf represents a new treatment option for patients with RRMM. As with other ADCs, MECs were observed and additional research is warranted to determine their pathophysiology. A multidisciplinary approach, involving close collaboration between eye care professionals and hematologist/oncologists, is needed to determine appropriate diagnosis and management of these patients.

Trial Registration: ClinicalTrials.gov Identifier, NCT03525678.

Keywords: Antibody-drug conjugate; Belantamab mafodotin; Cornea; In vivo confocal microscopy; Microcyst-like epithelial changes; Monomethyl auristatin F; Multiple myeloma; Oncology

\section{Key Summary Points}

Corneal microcyst-like epithelial changes (MECs) are frequently associated with antibody-drug conjugates (ADCs) containing monomethyl auristatin F, which have been studied in a variety of cancers, including belantamab mafodotin (belamaf, GSK2857916) currently being investigated for the treatment of relapsed or refractory multiple myeloma.

In this report, the authors further characterize belamaf-associated MECs and provide a representative case report. MECs typically are seen early in treatment, are manageable with dose modifications, and tend to resolve after completing treatment.

Presented images demonstrate that MECs appear as bilateral, diffuse, microcyst-like lesions on slit lamp photography; however, on confocal microscopy the affected areas appear as hyperreflective material that is (at least predominantly) intracellular.

Available literature on corneal events with other ADCs supports the observations with belamaf treatment and confirms the need for additional research on the underlying pathophysiology and optimal management.

The authors propose a mechanism whereby MECs represent an off-target effect of belamaf in the cornea leading to apoptosis of epithelial cells, which are eventually replaced with new epithelial cells, leading to the eventual resolution of MECs and symptoms after completing treatment. 


\section{DIGITAL FEATURES}

This article is published with digital features, including a summary slide, infographic and plain language summary, to facilitate understanding of the article. To view digital features for this article go to https://doi.org/10.6084/ m9.figshare.12326546.

\section{INTRODUCTION}

Multiple myeloma (MM) is an incurable hematologic cancer characterized by the uncontrolled accumulation of malignant plasma cells in the bone marrow $[1,2]$. It has an incidence rate of approximately 6.9 per 100,000 persons in the USA, with a median age at diagnosis of 69 years [3]. It typically has a poor prognosis; the median overall survival is less than 7 years for patients with Revised International Staging System stage II disease, and 3.6 years for stage III disease [4]. Despite recent advances in the management of $\mathrm{MM}$, there are a substantial number of patients who develop relapsed or refractory MM (RRMM) and are resistant to current standard-of-care options (proteasome inhibitors, immunomodulatory agents, and monoclonal antibodies [mAbs]) [5]. Evidence suggests that this patient population has a particularly unfavorable prognosis with currently available treatments (median progression-free survival: 3.4 months and median overall survival: 9.3 months) and represents an unmet medical need [6].

Belantamab mafodotin (belamaf; GSK2857916) is a first-in-class antibody-drug conjugate (ADC, also known as an immunoconjugate) targeting B-cell maturation antigen (BCMA) that is currently under clinical development for RRMM [7-9]. It is composed of an afucosylated humanized immunoglobulin G1 anti-BCMA mAb conjugated to a cytotoxic payload, the microtubule-disrupting agent known as monomethyl auristatin F (MMAF; Fig. 1) [10]. BCMA is a cell membrane receptor that is expressed on all malignant plasma cells and is essential for their proliferation and survival [2]. Belamaf binds to BCMA and kills MM cells via a multimodal mechanism involving, in part, delivery of MMAF to BCMA-expressing

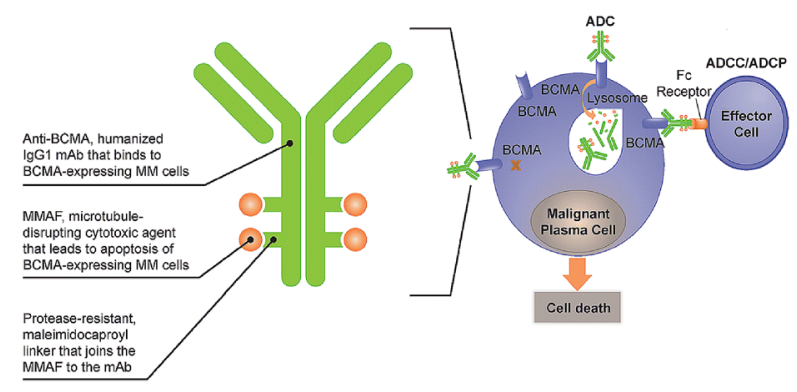

Fig. 1 Belamaf structure and mechanism of action. ADC antibody-drug conjugate, ADCC/ADCP antibody-directed cellular cytotoxicity/phagocytosis, BCMA B-cell maturation antigen, IgG1 immunoglobulin G1, mAb Monoclonal antibody, MM multiple myeloma, MMAF monomethyl auristatin $\mathrm{F}$

MM cells, thereby inducing apoptosis $[10,11]$. In both phase I (DREAMM-1; NCT02064387) and phase II (DREAMM-2; NCT03525678) studies, single-agent belamaf demonstrated clinically meaningful anti-myeloma activity in patients with heavily pretreated RRMM [7-9]. Specifically, in DREAMM-2, the overall response rate was $32 \%$ and the median duration of response was 11.0 months (95\% CI $4.2-\mathrm{NR}$ ) with a median follow-up of 12.4 months with belamaf $2.5 \mathrm{mg} / \mathrm{kg}$, the recommended dose for future development [12].

Many patients who received belamaf in these clinical studies developed changes within the corneal epithelium (which, in this report, we call microcyst-like epithelial changes, or MECs), similar to those observed with other MMAFcontaining ADC [7-9, 13]. Here, we report characteristics of these MECs and associated examination results observed in patients receiving the $2.5-\mathrm{mg} / \mathrm{kg}$ dose of belamaf in DREAMM-2 and provide a representative case. We also report the findings of a literature review on corneal changes associated with ADCs. Finally, we provide a proposed mechanism underlying these events, along with a screening and management paradigm for these patients.

\section{METHODS}

The full methodological details of DREAMM-2 were previously reported [7]. In brief, eligible patients with RRMM were randomized (1:1) to 
receive belamaf 2.5 - or $3.4-\mathrm{mg} / \mathrm{kg}$ every 3 weeks by intravenous infusion over $30 \mathrm{~min}$ or longer on day 1 of each cycle. Patients received treatment until disease progression or an unacceptable toxicity occurred. Patients without current corneal epithelial disease, with the exception of mild dry eye disease, were permitted to enroll. Here, we report data for patients receiving the $2.5 \mathrm{-mg} / \mathrm{kg}$ dose of belamaf (13-month followup), which was selected as the recommended dose for future studies on the basis of its similar anti-myeloma activity with a more favorable safety profile when compared with the $3.4-\mathrm{mg} /$ $\mathrm{kg}$ dose.

To ensure a comprehensive understanding of belamaf-associated corneal events in DREAMM2 , data were collected through corneal examinations and patient-reported ocular symptoms. Corneal examinations were conducted at baseline, prior to each treatment cycle, and every 3 weeks by an ophthalmologist or optometrist [7]. Corneal examinations included, at minimum, a slit lamp examination to identify changes in the cornea and a best-corrected visual acuity (BCVA) assessment to detect changes from baseline. These objective corneal examination findings observed on slit lamp microscopy (termed here as MECs) and BCVA results were combined and graded on the basis of the keratopathy and visual acuity (KVA) scale, protocol-specified criteria, and were used to guide dose modifications (including delays and reductions; Table 1). When the eye care professional recorded a corneal examination finding, the hematologist/oncologist reported an adverse event (AE) of microcyst-like epithelial keratopathy. Microcystlike epithelial keratopathy events were mapped to the preferred-term keratopathy per Medical Dictionary for Regulatory Activities (MedDRA). Patient-reported ocular symptoms (e.g., blurred vision and dry eye) were collected by the hematologist/oncologist. These patient-reported symptoms were recorded as AEs, and coded using MedDRA preferred terms. Both patient-reported symptoms and keratopathy were graded per the Common Terminology Criteria for Adverse Events version 4.03 (CTCAE v4.03) using the "Eye disorders, Other" criteria [14].

Per protocol, all MEC events were followed by the eye care professional until full resolution of ophthalmic changes or recovery to baseline. Recovery was defined as an event that was deemed clinically stable by the eye care professional. Clinical stability was defined as any grade 1 exam finding (per KVA scale) or no exam finding, and either a one-line decline in vision or no change in vision when compared with baseline. For patient-reported symptoms, recovery was defined as full recovery or return to baseline.

Patients were instructed to self-administer prophylactic corticosteroid eye drops (prednisolone acetate $1 \%$, prednisolone sodium phosphate $1 \%$, dexamethasone $0.1 \%$, or equivalent, one drop, four times daily [QID], starting 1 day predose, for a total of 7 days) and preservative-free lubricant eye drops (one drop, 4-8 times daily, beginning on cycle 1 , day 1 until end of treatment) in both eyes [7]. Because no specific inflammatory nature of ocular changes was identified in DREAMM-1, an ocular substudy $(n=30)$ was implemented in DREAMM-2 to determine the utility of corticosteroid eye drops. In the ocular sub-study, patients were instructed to administer corticosteroid eye drops in one eye only and preservative-free lubricant eye drops in both eyes for cycles 1-4. Patients were prohibited from wearing contact lenses for vision correction during the study. Bandage contact lens use was permitted under the management of an eye care professional. Belamaf dose modifications (delays and reductions) were permitted as part of the management strategy for MECs based on a combination of eye examination findings and change in BCVA from baseline.

A logistic regression analysis was conducted to determine possible risk factors for developing corneal events per KVA scale in patients with and without a history of dry eye at baseline. To assess the duration of time for patients to recover from keratopathy after treatment discontinuation, a post hoc, time-to-event (recovery) analysis was performed. Patients who did not recover as of last follow-up were censored at the last examination date in this analysis. Death before recovery was considered a competing risk event that precluded the observation of full recovery.

A literature search was performed on PubMed using the following search terms: "antibody-drug conjugate AND cornea OR eye OR 
Table 1 Recommended belamaf dose modifications based on eye examination findings per the KVA scale

Eye examination findings per KVA scale

Grade 1 Corneal examination finding(s)

Mild superficial keratopathy ${ }^{a}$

Change in $\mathrm{BCVA}^{\mathrm{b}}$

Decline from baseline of 1 line on Snellen

Visual Acuity

Grade 2 Corneal examination finding(s)

Moderate superficial keratopathy ${ }^{c}$

Change in $\mathrm{BCVA}^{\mathrm{b}}$

Decline from baseline of 2 or 3 lines (and

Snellen Visual Acuity not worse than 20/200)

Grade 3 Corneal examination finding(s)

Severe superficial keratopathy ${ }^{\mathrm{d}}$

Change in $\mathrm{BCVA}^{\mathrm{b}}$

Decline from baseline by more than 3 lines

(and Snellen Visual Acuity not worse than

20/200)

Grade 4 Corneal examination finding(s)

Corneal epithelial defect ${ }^{\mathrm{e}}$

Change in $\mathrm{BCVA}^{\mathrm{b}}$

Snellen Visual Acuity worse than 20/200
Recommended dose modifications

Continue treatment at current dose

Withhold treatment until improvement in both corneal examination findings and changes in BCVA to Grade 1 or better and resume at same dose

Withhold treatment until improvement in both corneal examination findings and changes in BCVA to Grade 1 or better and resume at a reduced dose

Consider treatment discontinuation for a Grade 4 event.

Based on a benefit:risk assessment, if continuing treatment with belamaf is being considered, treatment may be resumed at a reduced dose after the event has improved to Grade 1 or better event

$B C V A$ best-corrected visual acuity, $K V A$ keratopathy and visual acuity, $M E C$ microcystic-like epithelial change

DREAMM-2 utilized a pre-specified scale, the KVA scale, that combined slit lamp examination findings (e.g., keratopathy/ MECs) with an assessment of BCVA using Snellen Chart

a Mild superficial keratopathy (documented worsening from baseline), with or without symptoms

${ }^{b}$ Changes in visual acuity due to treatment-related corneal findings

${ }^{c}$ Moderate superficial keratopathy with or without patchy microcyst-like deposits, sub-epithelial haze (peripheral), or a new peripheral stromal opacity

d Severe superficial keratopathy with or without diffuse microcyst-like deposits involving the central cornea, sub-epithelial haze (central), or a new central stromal opacity

${ }^{\mathrm{e}}$ Corneal epithelial defect such as corneal ulcers

ocular OR keratopathy"; "immunoconjugate AND cornea OR eye OR ocular OR keratopathy". Additionally, the terms "antibody-drug conjugate" and "immunoconjugate" were used to find publications on corneal AEs associated with this class of drugs. Based on the results of these parameters, additional searches were conducted with the following terms: "macropinocytosis AND cornea OR eye OR ocular OR keratopathy"; "prevent macropinocytosis"; "inhibit macropinocytosis."

This study was performed in accordance with the Declaration of Helsinki and Good Clinical Practice guidelines following approval by the ethics committees and institutional review boards at each study site (listed within the acknowledgements). All patients provided written informed consent prior to enrollment. 


\section{RESULTS}

In DREAMM-2, MECs as seen on slit lamp microscopy comprised the most common AE reported in patients receiving belamaf. At the recommended belamaf dose $(2.5 \mathrm{mg} / \mathrm{kg}$, $n=95), 72 \%(n=68)$ of patients in DREAMM-2 developed MECs (Table 2).

Table 2 Incidence, duration, and resolution of MECs, BCVA change, and corneal symptoms in patients receiving belamaf $(2.5 \mathrm{mg} / \mathrm{kg})$ in DREAMM-2

\begin{tabular}{|c|c|c|c|c|c|}
\hline & \multicolumn{3}{|c|}{ Eye examination findings per KVA scale } & \multicolumn{2}{|l|}{ CTCAE scale } \\
\hline & $\begin{array}{l}\text { MECs } \\
(n=95)\end{array}$ & $\begin{array}{l}\text { BCVA change } \\
(n=95)\end{array}$ & $\begin{array}{l}\text { MECs + BCVA } \\
\text { change }(n=95)\end{array}$ & $\begin{array}{l}\text { Blurred vision } \\
(n=95)\end{array}$ & $\begin{array}{l}\text { Subjective } \\
\text { dry eye } \\
(n=95)\end{array}$ \\
\hline Any grade, $n(\%)^{\mathrm{a}}$ & $68(72)$ & $51(54)$ & $68(72)$ & $24(25)$ & $14(15)$ \\
\hline \multicolumn{6}{|l|}{ Maximum grade } \\
\hline Grade 1 & $8(8)$ & $7(7)$ & $7(7)$ & $11(12)$ & $9(9)$ \\
\hline Grade 2 & $16(17)$ & $15(16)$ & $14(15)$ & $9(9)$ & $4(4)$ \\
\hline Grade 3 & $43(45)$ & $28(29)$ & $45(47)$ & $4(4)$ & $1(1)$ \\
\hline Grade 4 & $1(1)$ & $1(1)$ & $2(2)$ & 0 & 0 \\
\hline $\begin{array}{l}\text { Median time to onset of first } \\
\text { occurrence (range), days }\end{array}$ & $\begin{array}{l}37.0 \\
\quad(19-143)^{\mathrm{b}}\end{array}$ & $64.0(20-213)$ & $36.0(19-143)$ & $51.5(6-339)$ & $\begin{array}{l}42.0 \\
\quad(12-151)\end{array}$ \\
\hline $\begin{array}{l}\text { Median duration of first event } \\
\text { (range), days }\end{array}$ & $\begin{array}{l}86.5 \\
\quad(8-358)^{\mathrm{b}}\end{array}$ & $33.0(8-127)^{\mathrm{b}}$ & $96.0(8-358)^{\mathrm{b}}$ & $42.5(6-441)$ & $\begin{array}{l}39.0 \\
\quad(12-316)\end{array}$ \\
\hline \multicolumn{6}{|l|}{ First event outcomes, ${ }^{c} n / N(\%)$} \\
\hline Recovered & $46 / 60(77)^{\mathrm{b}}$ & $34 / 44(77)^{\mathrm{b}}$ & $45 / 61(74)^{b}$ & $16 / 24(67)$ & $12 / 14(86)$ \\
\hline Not recovered & $14 / 60(23)^{b}$ & $10 / 44(23)^{\mathrm{b}}$ & $16 / 61(26)^{b}$ & $8 / 24(33)$ & $2 / 14(14)$ \\
\hline \multicolumn{6}{|c|}{ Event outcomes as of last follow-up, ${ }^{c} n / N(\%)$} \\
\hline Recovered & $29 / 60(48)^{b}$ & $26 / 44(59)^{\mathrm{b}}$ & $30 / 61(49)^{\mathrm{b}}$ & $15 / 24(63)$ & $11 / 14(79)$ \\
\hline Not recovered & $31 / 60(52)^{b}$ & $18 / 44(41)^{\mathrm{b}}$ & $31 / 61(51)^{\mathrm{b}}$ & $9 / 24(38)$ & $3 / 14(21)$ \\
\hline Dose delays due to event, $n(\%)$ & - & - & $45(47)^{\mathrm{d}}$ & $7(7)^{\mathrm{e}}$ & $3(3)$ \\
\hline $\begin{array}{l}\text { Dose reductions due to event, } \\
n(\%)\end{array}$ & - & - & $24(25)^{\mathrm{d}}$ & $2(2)^{\mathrm{e}}$ & 0 \\
\hline
\end{tabular}

Safety population $(n=95)$ defined as all patients who received $\geq 1$ dose of belamaf

$B C V A$ best-corrected visual acuity, CTCAE v4.03 Common Terminology Criteria for Adverse Events version 4.03, KVA keratopathy and visual acuity, $M E C s$ microcyst-like epithelial changes

${ }^{a}$ MEC and BCVA change grade based on KVA scale (Table 1) with MEC + BCVA grade based on the highest grade for either MECs or BCVA change per KVA scale. Blurred vision and dry eye graded based on CTCAE v4.03

${ }^{\mathrm{b}}$ Data for $\geq$ grade 2 events per KVA scale

c Recovery of MECs was defined as an event that was deemed clinically stable by the eye care professional. Clinical stability was defined as any grade 1 exam finding (per KVA scale) or no exam finding, and either a one-line decline in vision or no change in vision when compared with baseline

${ }^{d}$ Dose delays and reductions were based on the grade for MECs + BCVA change

${ }^{\mathrm{e}}$ Included preferred terms vision blurred, visual acuity reduced, visual impairment, diplopia 
Grade 1 (mild) MECs occurred in 8\% of patients, grade 2 (moderate) MECs occurred in $17 \%$, grade 3 and grade 4 MECs (severe) occurred in $45 \%$ and $1 \%$ of patients, respectively (Table 2). One patient (with a history of cataract surgery in the right eye) developed a central corneal ulcer that resolved 9 days after onset with the use of topical antibiotics.

The median time to onset of MECs was 37.0 days (Table 2). Accordingly, they were first observed after the first dose in $25 \%$ of patients. Progressively more patients developed MECs with each dose, with $69 \%$ of patients experiencing their first event by dose 4 and only 2 patients developing a corneal event for the first time after dose 4 . MECs comprised the most common AE leading to belamaf dose modification, accounting for $47 \%$ of dose delays and
$25 \%$ of dose reductions. In total, $3(3 \%)$ patients discontinued treatment because of corneal events (1 [1\%] each due to MECs, blurred vision, and reduced BCVA).

As of the data cutoff, $77 \%(46 / 60)$ of patients had recovered from their first occurrence of grade $\geq 2$ MECs. The median time to resolution of MECs for the first event was 86.5 days in all patients, including those with and without dose modifications. Forty-two (44\%) patients had grade $\geq 2$ corneal examination findings at the time of treatment discontinuation or had an onset post-treatment discontinuation. Of those, it is estimated that the majority (80\%) would recover with continued follow-up (Fig. 2). Peak recovery period was approximately $2-6$ months post-treatment. Additional recoveries were

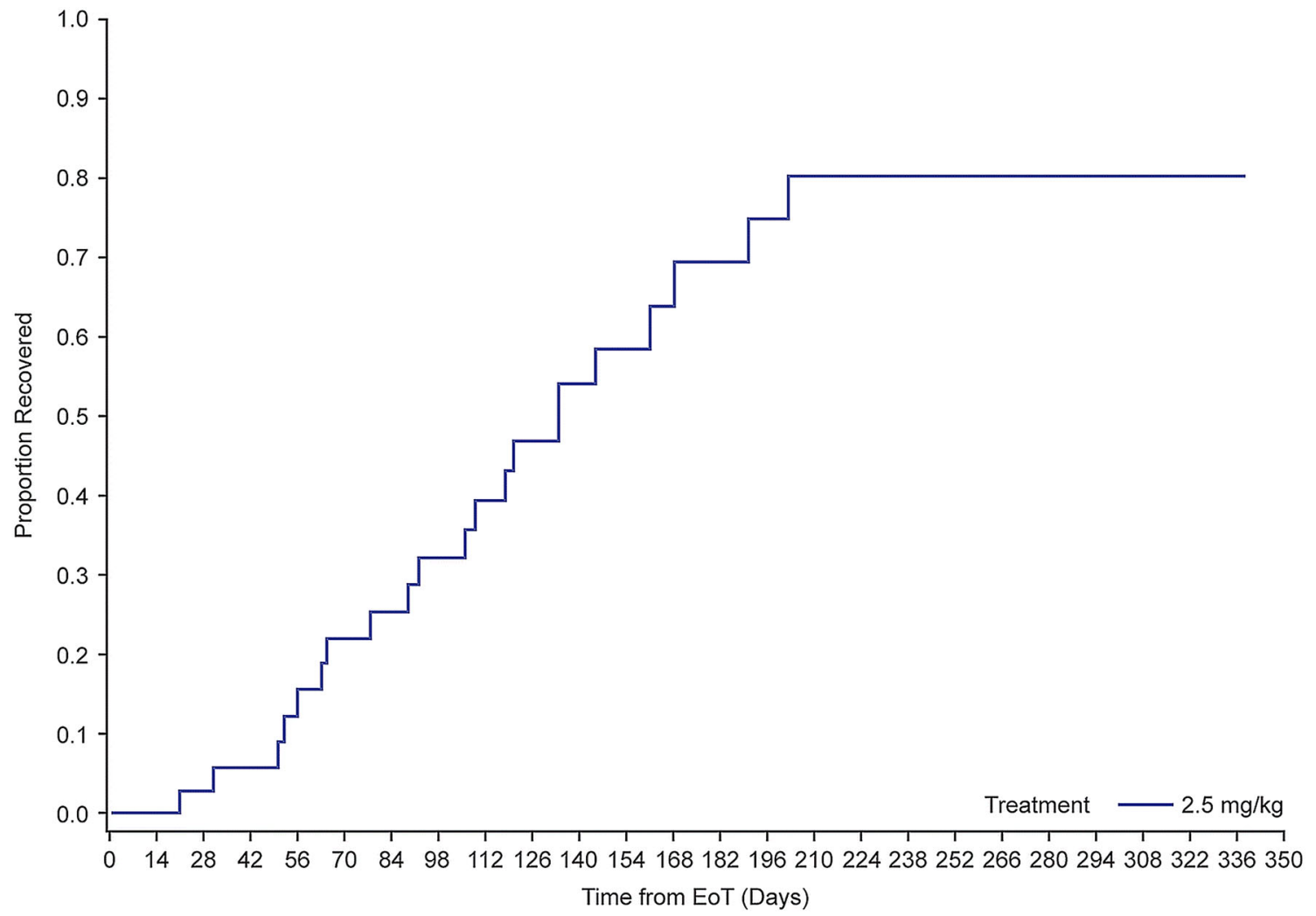

Fig. 2 Time-to-recovery analysis for MECs in patients receiving belamaf $(2.5 \mathrm{mg} / \mathrm{kg})$ in DREAMM-2. EoT end of treatment, MEC microcyst-like epithelial changes. EoT defined as the last dose date (for patients with an unresolved event at treatment discontinuation) or the event onset date (for patients with an event that started post treatment discontinuation due to any reason). Analysis included patients who were not recovered at EoT exposure (defined as 20 days after the last infusion) and those who had events that started after treatment discontinuation $(n=39)$. Twenty-one percent $(8 / 39)$ of patients died before recovery 
observed in a smaller number of patients after that.

Examination findings were not always accompanied by either patient symptoms or changes in BCVA. Among patients with MECs, $72 \%$ reported symptoms (including blurred vision or subjective dry eye) and/or had a decrease in BCVA ( $\geq 2$ lines decline in the better-seeing eye). Common patient-reported symptoms overall included blurred vision (25\%) and subjective dry eye (15\%; Table 2). Blurred vision and dry eye events were mainly grade 1 (12\% and $9 \%$, respectively) or grade 2 (9\% and $4 \%$, respectively) per CTCAE v4.03. The median time to the first occurrence of blurred vision and dry eye symptoms was 51.5 and 42.0 days and the median duration was 42.5 and 39.0 days, respectively. As of the latest followup, $63 \%$ of patients with blurred vision and $79 \%$ with dry eye had recovered. Of note, $19 \%$ of patients reported a history of dry eye prior to starting treatment. These patients were statistically more prone to developing corneal events per the KVA scale compared with patients who did not have a history of dry eye $(p=0.02)$.
Eighteen percent of patients experienced a decline in BCVA to $20 / 50$ or worse in their better-seeing eye at least once during or after the treatment period. As of the last follow-up, $82 \%$ of patients recovered (BCVA of $20 / 40$ or better; Table 3). The median duration of this decline in BCVA was 21.5 days; therefore, most patients recovered after one assessment interval (conducted every 21 days). Overall review of the worst BCVA measurement during the study period revealed that $28 \%(25 / 88)$ of patients showed a doubling of the minimum angle of resolution in their better-seeing eye (logMAR change $\geq 0.3$ to $<0.6$ ).

One patient experienced a worsening of BCVA to $20 / 200$ in their better-seeing eye (BCVA was worse than 20/400 in the other eye at baseline) that recovered to baseline during follow-up. Two (2\%) patients experienced severe vision loss (logMAR change $\geq 0.6$ in the better-seeing eye). Both resolved (improved to logMAR change $<0.6$ ) after 22.0 days (one assessment interval).

Table 3 Clinically meaningful changes in BCVA, time to onset, and time to resolution in patients receiving belamaf $(2.5 \mathrm{mg} / \mathrm{kg})$ in DREAMM-2

\begin{tabular}{lll}
\hline & $\begin{array}{l}\text { BCVA of 20/50 or worse in the } \\
\text { better-seeing eye }(\boldsymbol{n}=\mathbf{9 5})^{\mathbf{a}}\end{array}$ & $\begin{array}{l}\text { BCVA of 20/200 or worse in the } \\
\text { better-seeing eye }(\boldsymbol{n}=\mathbf{9 5})^{\mathbf{b}}\end{array}$ \\
\hline$n(\%)$ & $17(18)$ & $1(1)$ \\
Median time to onset (range), days & $66.0(20-442)$ & $21.0(21-21)$ \\
Event outcomes as of last follow-up, $n / N(\%)$ & & $1 / 1(100)^{\mathbf{d}}$ \\
$\quad \begin{array}{l}\text { Recovered } \\
\quad \text { Not recovered }\end{array}$ & $14 / 17(82)^{\mathbf{c}}$ & 0 \\
$\begin{array}{l}\text { Median time to resolution in patients who } \\
\text { recovered as of last follow-up (range), days }\end{array}$ & $n=14(18)$ & $n=1$ \\
\hline
\end{tabular}

Safety population $(n=95)$ defined as all patients who received $\geq 1$ dose of belamaf. Data represent BCVA changes in the patients' better-seeing eye

$B C V A$ best-corrected visual acuity

a Better than 20/50 at baseline in the better-seeing eye and 20/50 or worse post baseline in the better-seeing eye

b Better than 20/200 at baseline in the better-seeing eye and 20/200 or worse post baseline in the better-seeing eye

c Recovery was defined as 20/40 or better in the better-seeing eye

d Recovery was defined as better than 20/200 in the better-seeing eye 

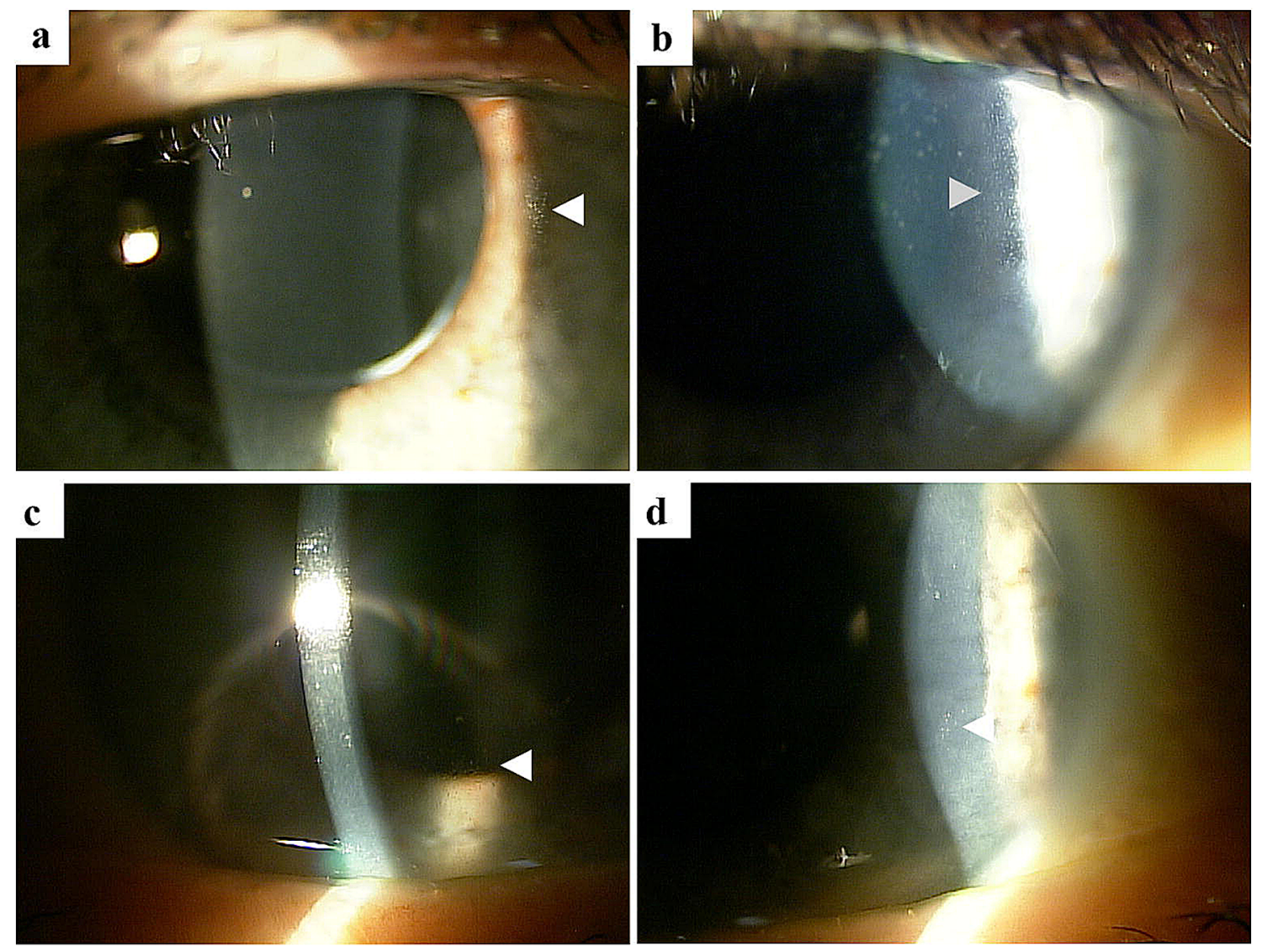

Fig. 3 Representative slit lamp microscopic images of MECs. MECs microcyst-like epithelial changes. Slit lamp microscopic images (a-d) demonstrating MECs (see arrowheads). The lesions are small, located within the

\section{Description of MECs and Imaging Findings}

MECs were described as bilateral, diffuse lesions on slit lamp microscopic images (Fig. 3). Subjective and objective changes in vision in patients receiving belamaf appeared to correspond to the location of the MECs; more central changes (i.e., those within the visual axis) observed in a few patients tended to correlate with blurred vision as compared with mid-peripheral changes observed over time in some patients (i.e., those outside of the visual axis). On in vivo confocal microscopy (IVCM), the involved areas of the corneal epithelium were found to contain hyperreflective material, rather than microcysts (Fig. 4).

corneal epithelium, and are seen here in the corneal periphery and mid-periphery. Visualization requires high magnification and is aided by the use of retroillumination off the iris or indirect illumination

MECs appear clinically distinct from other pathologies that are commonly encountered by corneal specialists, including Cogan microcysts (lesions are more opaque, larger, and have varied shapes), corneal amyloidosis (typically present within the stroma), and band keratopathy (calcium deposition involving Bowman layer typically resulting in an opaque confluent "band").

However, similar descriptions and images of these slit lamp microscopy and IVCM findings have been reported in studies and case reports with other ADCs (see "Literature Review of Corneal Changes with ADCs" section for additional details) [13, 15-20]. 


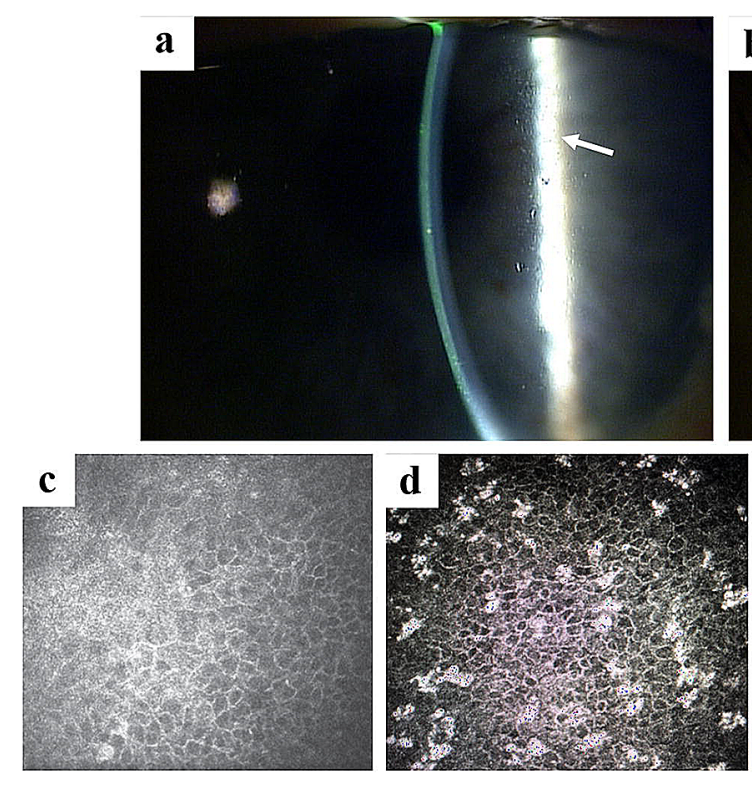

Fig. 4 Case report representative slit lamp and in vivo confocal microscopic images of MECs. MECs microcystlike epithelial changes. Slit lamp microscopic images of the right (a) and left (b) eyes, demonstrating MECs (arrows). These lesions are best visualized at medium to high magnification. Retroillumination or indirect illumination can be helpful. In vivo confocal microscopic image from

\section{Representative Case}

This case is from a 74-year-old male patient diagnosed with MM 5 years prior to enrolling in DREAMM-2 who had progressive disease following five prior lines of anti-myeloma therapy. He had previously been diagnosed with dry eye disease and had undergone uneventful cataract surgery in both eyes. He was enrolled into the belamaf $2.5-\mathrm{mg} / \mathrm{kg}$ arm of the study and participated in the ocular sub-study, in which he was instructed to administer corticosteroid eye drops to only one eye for cycles 1-4.

At the time of screening, he had mild punctate fluorescein staining in both corneas, a small $(1 \mathrm{~mm})$ peripheral Salzmann nodule OS, and a BCVA of 20/20 OU. Per protocol, he administered preservative-free lubricant eye drops QID in both eyes throughout the study and corticosteroid eye drops (prednisolone sodium phosphate 1\%) QID in one eye only during treatment cycles $1-4$, then in both eyes for the
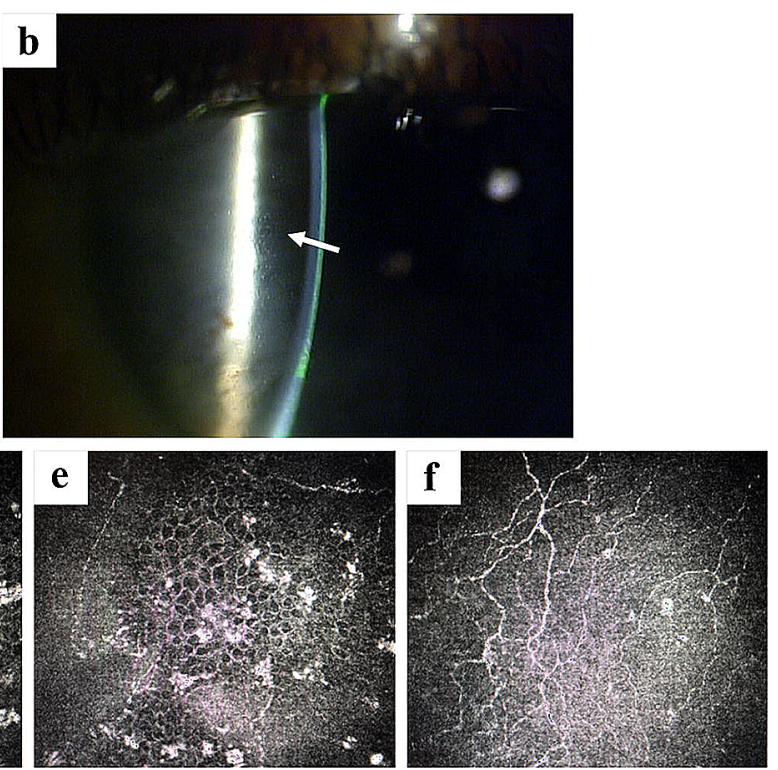

the same patient $(\mathbf{c}-\mathbf{f})$ demonstrating hyperreflective opacities within the corneal epithelium. These opacities were noted to be most prominent in the wing cells (d) and basal cells (e), as compared with the superficial cells (f). They were not visualized within the anterior stroma $(\mathbf{f})$ or endothelium (not shown)

remaining treatment cycles (starting 1 day predose, for a total of 7 days).

Following the first dose of belamaf $2.5 \mathrm{mg} / \mathrm{kg}$, he presented on day 27 with MECs characterized as mild/patchy in the periphery/ mid-periphery on slit lamp microscopy (Fig. 4a, b). IVCM of the involved areas revealed hyperreflective opacities (Fig. 4c-f). The BCVA at this visit was 20/20 OU. The patient's dose was reduced to $1.9 \mathrm{mg} / \mathrm{kg}$ after cycle 1 to manage an $\mathrm{AE}$ (platelet count decrease). MECs and BCVA remained stable over the next eight examinations conducted between days 40 and 174 .

After the eighth dose of belamaf (administered on day 195), an increased number of MECs was noted OU, as well as $3+$ punctate staining with fluorescein, and areas of whorllike staining OU (Fig. 5). Treatment was held, and he was instructed to use corticosteroid drops QID OU, and preservative-free lubricant eye drops QID OU, for 1 month. At the time of the next examination, the MECs and whorl-like staining had completely resolved. The topical 


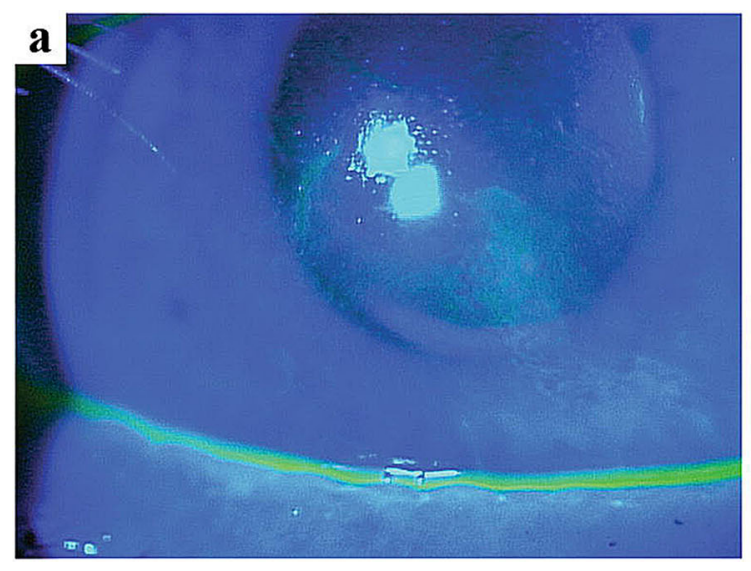

Fig. 5 Case report representative high-magnification fluorescein staining slit lamp microscopic images. Broadbeam slit lamp microscopic images with cobalt blue light demonstrating fluorescein staining of the right (a) and left

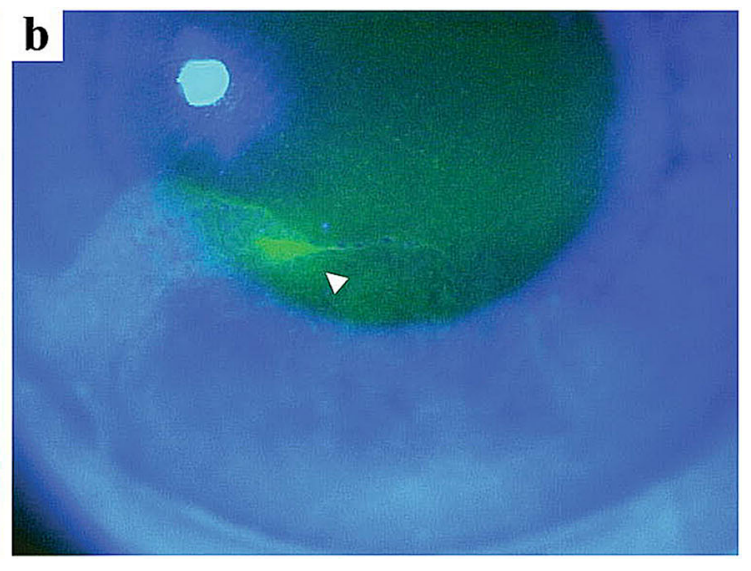

(b) eyes. Whorl-like staining was noted in both corneas, most prominent in the inferonasal quadrant of the left eye (arrowhead)

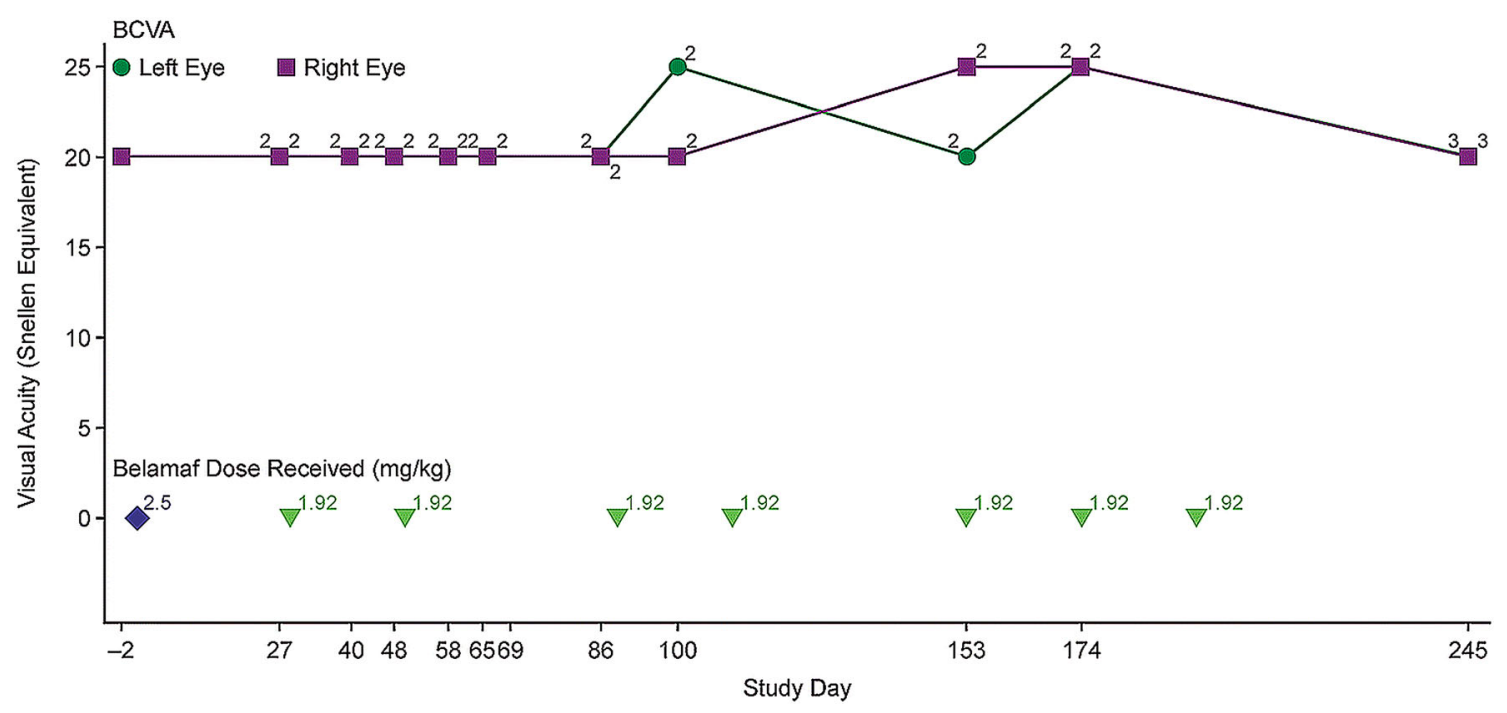

Fig. 6 Change in BCVA over time with belamaf treatment in the DREAMM-2 case report. BCVA bestcorrected visual acuity, KVA keratopathy and visual acuity, MECs microcyst-like epithelial changes. Corneal

eyedrops were tapered, and he was restarted on the study drug at the $1.9-\mathrm{mg} / \mathrm{kg}$ dose.

As of this report, he had received eight doses of belamaf (one dose at $2.5 \mathrm{mg} / \mathrm{kg}$ and seven doses at $1.9 \mathrm{mg} / \mathrm{kg}$; total cumulative dose $15.94 \mathrm{mg} / \mathrm{kg}$; Fig. 6). The patient's best clinical response was a very good partial response (per International Myeloma Working Group criteria [21]). examination finding (MECs and changes in BCVA) were graded per KVA scale (Table 1), and represented (as grades 1-4) above each line for each examination

\section{Literature Review of Corneal Changes with ADCs}

\section{Incidence and Description}

In a literature search for ADCs and ocular changes, 145 published manuscripts were identified and 28 were related to ocular AEs with ADCs in clinical trials (all in patients with 
cancer). A review of these 28 studies and case reports revealed that ADCs directed against other targets have also demonstrated a high incidence of ocular surface AEs. The terminology used to describe these events varied among reports; however, several of these terms (e.g., "microcyst-like corneal epitheliopathy," "microcystic epitheliopathy," "corneal epithelial microcyst") suggest these events have a microcyst-like presentation in the corneal epithelium, consistent with the MECs described here with belamaf treatment $[16,22,23]$. Ocular surface AEs observed with ADCs have typically been described as multiple, bilateral, microcyst-like lesions in the superficial cornea [17-19]. In some reports, MECs were noted to start in the periphery and migrate centrally toward the pupil [16, 17, 19, 24]. Overall, ocular surface changes observed and the methods for assessing these events in ADC clinical trials are often not well described [13]. Further, given the limited available reports and interstudy variation in patient populations and other treatment-related factors, it is difficult to draw conclusions about the incidence and distinguishing features of these events with ADCs [13].

Prior to this report, only a few publications have provided slit lamp microscopic images or IVCM images of MECs; each of these demonstrated similar observations to those reported here [15-20]. In a case report of a patient receiving trastuzumab emtansine (an ADC with a non-MMAF payload) for breast cancer, corneal changes were described as multiple, intraepithelial spherical lesions in the midperiphery on slit lamp microscopy [15]. Hyperreflective lesions were observed on IVCM $[15,16,18]$. A second case report demonstrated similar findings but was later retracted as a result of patient unmasking [16, 25].

Evidence suggests the cytotoxic payload of an ADC is strongly associated with the corneal events observed, and MECs are most often associated with MMAF-containing ADCs like belamaf $[13,26]$. In clinical trials of other MMAF-containing ADCs (e.g., in non-Hodgkin's lymphoma, renal cell carcinoma, and glioblastoma), rates of ocular events ranged from $31 \%$ to $92 \%$, with grade $3 / 4$ events (per CTCAE grading) occurring in up to $35 \%$ of patients $[19,22,23,27-31]$. However, ocular surface AEs have also been observed with ADCs containing other cytotoxic payload agents including DM1 (e.g., emtansine and duocarmazine), DM4 (e.g., ravtansine and soravtansine), and less commonly with monomethyl auristatin E (MMAE; [e.g., vedotin]). Incidence rates of these findings varied: $24 \%$ with coltuximab ravtansine in lymphoma/leukemia; $24-48 \%$ with mirvetuximab soravtansine in ovarian cancer; $60 \%$ with tisotumab vedotin in solid tumors; and $71 \%$ with trastuzumab duocarmazine in breast cancer [32-38]. These events were mostly grade $1 / 2$ regardless of the ADC cytotoxic payload (MMAF or other) $[17,19,22,23,27-38]$. Overall, corneal events observed with ADCs are described as mild and reversible, and some are manageable with dose modifications and supportive care [13].

Common patient-reported symptoms observed with ADCs include subjective dry eye and blurred vision; however, the rates of these events seem to vary widely among ADC payloads. In reports of MMAF-containing ADCs, the incidence of dry eye symptoms ranged from $23 \%$ to $50 \%$, and the incidence of blurred vision ranged from $12 \%$ to $65 \%[19,22,23,27-31]$. For DM1 ADCs trastuzumab emtansine and trastuzumab duocarmazine, the incidence of dry eye symptoms was $11 \%$ and $31 \%$, respectively; blurred vision was only reported for trastuzumab duocarmazine (11\%) [32, 39]. Subjective dry eye occurred in 3-27\% of patients receiving DM4-containing ADCs and blurred vision occurred in $11-100 \%$ of patients $[17,24,34-38,40]$.

\section{Proposed Pathophysiology}

MMAF was selected as the cytotoxic payload in belamaf because of its potent anti-myeloma activity and, unlike MMAE, free, soluble MMAF is not toxic to surrounding normal cells [10]. Therefore, in the case of belamaf, which requires MMAF for its anti-myeloma effects, it is important to investigate the potential underlying mechanisms of ADC-associated corneal changes.

Although the pathophysiology of the MECs observed in patients enrolled in DREAMM-2 remains unclear, a review of data from other 
ADCs provides some clues. Corneal findings associated with ADCs may represent either an on-target or off-target mechanism of AEs $[13,41,42]$. In an on-target mechanism, noncancerous cells express the target antigen, leading to binding of the $\mathrm{mAb}$ and subsequent release of the cytotoxic payload into the cell $[41,42]$. One report described abnormal corneal lesions in a patient receiving trastuzumab emtansine, a DM1-containing ADC targeting HER2 developed for metastatic breast cancer [20]. Given that HER2 is expressed in the corneal epithelium, the authors attributed these corneal events to an on-target mechanism of the ADC [20, 43]. Alternatively, an off-target mechanism occurs when non-cancerous cells that do not express the target antigen are nevertheless exposed to the cytotoxic payload; this may occur via a number of mechanisms, including Fc-receptor-mediated endocytosis, pinocytosis (particularly macropinocytosis), and bystander toxicity in which the cytotoxic payload is prematurely cleaved from its linker $[41,42]$. Given the majority of the proteins targeted by these agents are not expressed in the cornea (e.g., BCMA), the MECs may primarily represent an off-target mechanism $[13,41,42,44,45]$.

Some authors have hypothesized that ADCs may reach the cornea through the vascularized area of the limbus $[16,44]$. Alternatively, ADCs may also reach the cornea through the tear film. Belamaf has been quantified in the plasma of animals and humans. It has also been detected, but was not quantifiable, in rabbit tears (collected using Schirmer strips) following single and repeat intravenous dosing for up to 4 weeks at 15 and $30 \mathrm{mg} / \mathrm{kg} /$ week (unpublished data). With either route, once they reach the cornea, ADCs may be internalized by progenitor cells via macropinocytosis, an off-target mechanism $[26,41,42,44,46]$. This non-specific form of cellular uptake is driven by actin polymerization at the plasma membrane, creating a vertical protrusion (ruffle) that collapses to create an enclosed cavity that is internalized as a vesicle [47]. Macropinocytosis has also been implicated as a mechanism for the development of thrombocytopenia via the apoptosis of megakaryocyte progenitor cells, an $\mathrm{AE}$ commonly reported with belamaf and other MMAF-containing ADCs [7, 26, 41, 42, 44, 48]. There is evidence that some ADCs may be internalized into the corneal epithelium via this endocytic pathway. For example, AGS-16C3F is an MMAF-containing ADC currently in development for the treatment of metastatic renal cell carcinoma [23]. Zhao et al. performed in vitro studies using a primary human corneal epithelial cell (HCEC) line as well as two other cell lines (fibroblasts and human umbilical vein endothelial cells) [44]. AGS-16C3F was found to be internalized by HCECs using immunostaining visualized via laser scanning in vitro confocal fluorescence microscopy. In comparing a modified structure of AGS-16C3F (with a reduced ability to undergo macropinocytosis) to the original molecule, there was significantly improved cell viability [44]. Furthermore, rabbits treated with the modified AGS-16C3F molecule had less severe and delayed ocular events compared with rabbits treated with the original molecule [44].

In a literature search for macropinocytosis and the eye, 15 published manuscripts were identified and 4 were related to macropinocytosis in the cornea (including the report by Zhao et al. [44]). It is hypothesized that corneal epithelial cells may use macropinocytosis as an alternative means of absorbing tear fluid and its nutrients and/or as a mechanism to reduce excessive stromal fluid subsequent to corneal epithelial injury [49]. Macropinocytosis is also a mechanism for internalization of indomethacin nanoparticles used to enhance the transcorneal penetration of some ophthalmic solutions [50]. Evidence suggests that viruses such as human adenovirus D37 associated with epidemic keratoconjunctivitis and bacteria such as Pseudomonas or Acanthamoeba may also utilize macropinocytosis to enter corneal epithelial cells, and that this mechanism may be enhanced in the setting of dry eye $[49,51]$. Pseudomonas and Acanthamoeba cluster near erythropoietin-producing hepatocellular A2 (EphA2) receptors on corneal epithelial cells and/or secrete proteins that activate EphA2, which induces macropinocytosis [49].

Once internalized, ADCs can inhibit cellular proliferation of HCECs [44]. Given the primary 
a Normal conditions

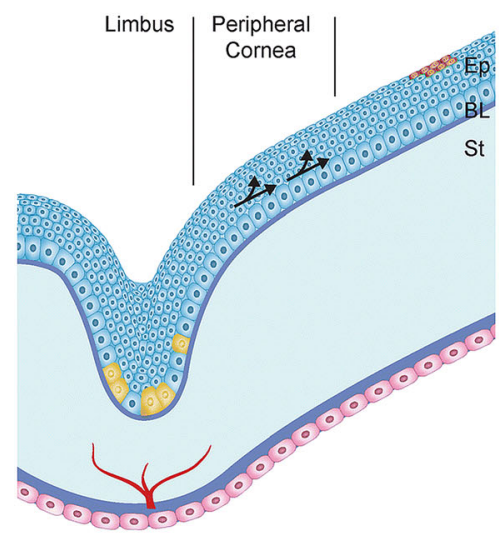

c Migration of early apoptotic corneal epithelial cells to peripheral cornea (MECs observed, but no symptoms) b Belamaf internalization: Belamaf enters the cornea, is internalized by limbal epithelial stem cells, and induces apoptosis

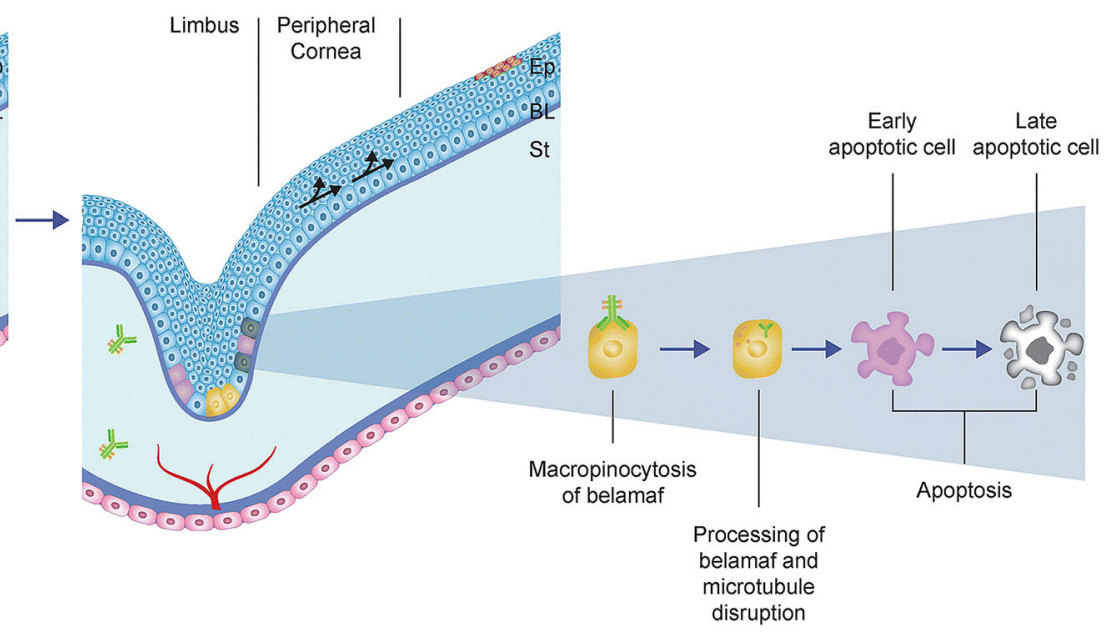

d Migration of early and late apoptotic corneal epithelial cells to central cornea (MECs observed and symptoms reported) e Migration of new corneal epithelial cells to replace cells that have undergone apoptosis (resolution of MECs and symptoms)

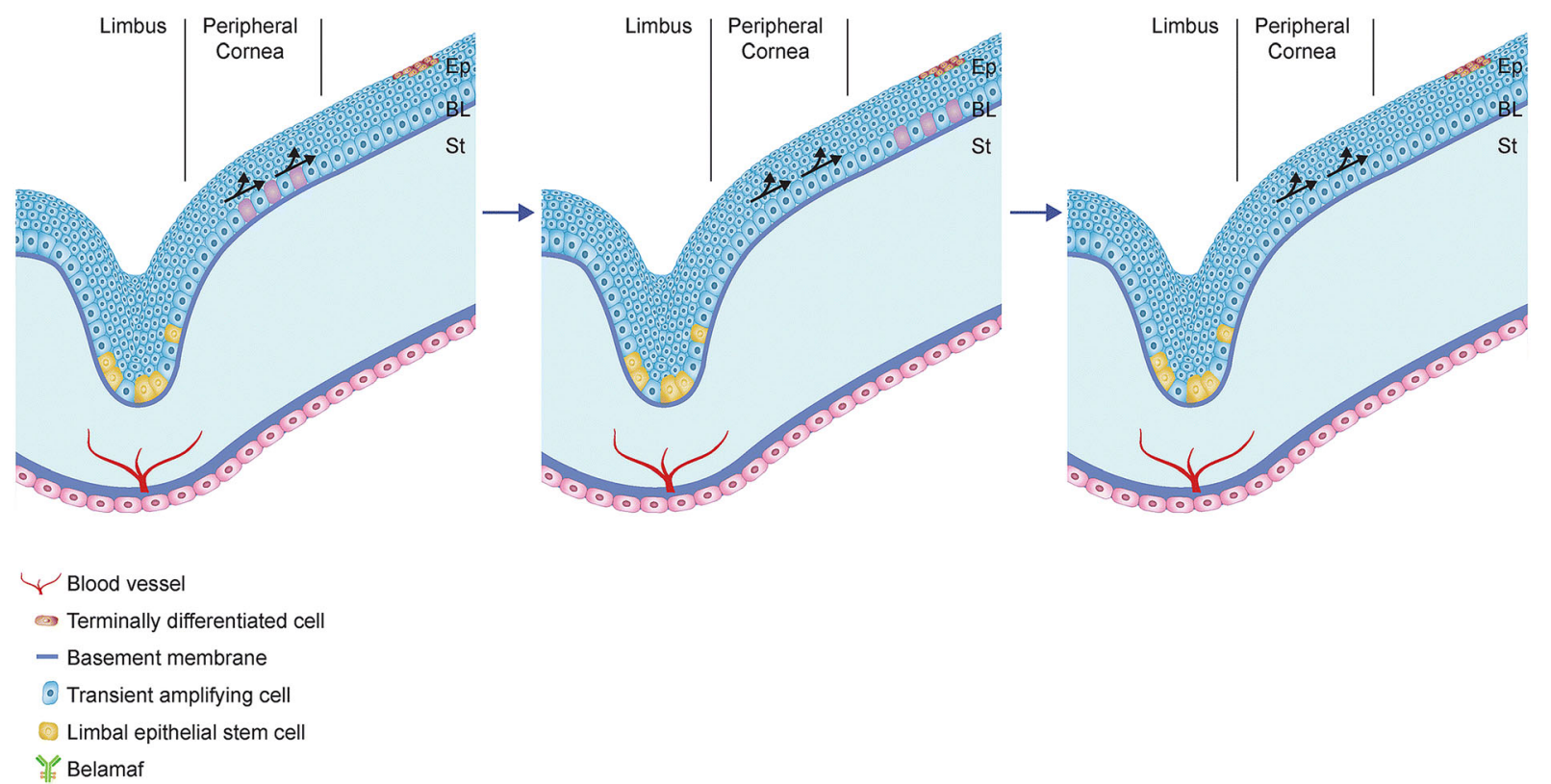

Fig. 7 Proposed mechanism of belamaf-associated MECs. Ep epithelium, BL Bowman's layer, MECs microcyst-like epithelial changes, St stroma

mechanism of action of belamaf and other ADCs (delivery of a cytotoxic, microtubule-inhibiting payload), these ADC-containing corneal epithelial cells may eventually undergo apoptosis [26]. Under normal conditions, daughter cells of actively dividing basal corneal epithelial cells migrate anteriorly as they differentiate into wing cells and terminally into superficial epithelial cells [52, 53]. Apoptotic cells are shed via desquamation [13]. The 
hyperreflective lesions observed on IVCM here and in previous ADC studies may represent ADC-containing corneal epithelial cells that have either not yet undergone apoptosis or are in a stage of apoptosis, prior to cell death $[15,16]$. In our cohort, these findings appear to be most prominent in the basal layer of the epithelium. An increase in dendritic cells near the subbasal nerve plexus has also been reported; the clinical significance of this finding is unclear [15]. In summary, we propose here the mechanism of MECs observed with belamaf treatment based on imaging from patients in DREAMM-2 and present descriptions and images of these events reported with other ADCs (Fig. 7). Upon systemic administration, belamaf enters the cornea, either through the vascularized region of the limbus or the tear film. It then becomes internalized by corneal epithelial cells in the basal layer (i.e., transient amplifying cells) through macropinocytosis, thereby initiating apoptosis. Belamaf-containing cells in various stages of apoptosis continue to migrate centrally and anteriorly, whereas cells that have completed apoptosis are extruded. These migrating belamaf-containing cells can be visualized by IVCM as hyperreflective opacities. As they migrate toward the visual axis, patientreported symptoms of blurred vision and BCVA changes occur. Over time, new epithelial cells are generated that replace the belamafcontaining cells that have undergone apoptosis. Preclinical and clinical investigations are ongoing to validate these steps of the proposed mechanism.

\section{Treatment Strategies}

While on-target mechanisms of AEs may pose a more significant challenge when treating patients with ADCs, off-target mechanisms may allow for mitigation strategies while maintaining drug efficacy. To our knowledge, inhibition of macropinocytosis as a means of mitigating the off-target effects of an ADC has not been reported in a clinical trial. While Zhao et al. reported the results of modifying the structure of ADC molecules to reduce their ability to undergo macropinocytosis [44], another theoretical approach would be to utilize a therapeutic agent that inhibits this process. A screen of US Food and Drug Administration (FDA)-approved compounds identified three drugs that strongly inhibited macropinocytosis without exerting cytotoxic effects or inhibiting endocytic pathways: imipramine, phenoxybenzamine, and vinblastine [54]. Of these, imipramine was shown to block membrane ruffle formation and inhibit macropinocytosis in macrophages, dendritic cells, and cancer cells, although it did not inhibit $\mathrm{Na}^{+} / \mathrm{H}^{+}$exchanger activity in macrophages [54]. These agents have not yet been tested for this indication in humans, so it is unclear if they may be a viable option to mitigate corneal changes observed with ADCs.

The use of prophylactic topical corticosteroid eye drops to mitigate ocular AEs (including MECs) has been reported with mixed effects; some studies support their use, although overall no clear benefit has been demonstrated $[7,19,22,24,27,31-33,38,55,56]$. However, these studies were not designed to evaluate the effectiveness of corticosteroid eye drops on mitigating MECs. One study prospectively examined the effects of prophylactic corticosteroid eye drops in patients treated with ADCs; this study examined 113 patients treated with mirvetuximab soravtansine and recommended prophylactic corticosteroid eye drops as a mitigation strategy, on the basis of a trend toward a lower incidence of keratopathy with prophylaxis, although the benefit was not statistically significant [38]. The use of corticosteroid eye drops showed some benefit in managing ocular events that occurred with the MMAF-containing ADCs ABT-414 and SGN-75 [19, 24, 27, 31]. Conversely, in DREAMM-2, corticosteroid eye drops were an ineffective prophylaxis for the development of changes to the corneal epithelium associated with belamaf treatment (please see "Recommended Monitoring, Diagnosis, and Management Techniques") [7].

Dose modifications have also been reported as a strategy to reduce the presence of MECs. Treatment-related corneal changes with belamaf were adequately managed with dose delays and reductions in DREAMM-2 [7]. This is supported by other studies that found that corneal changes improved or resolved upon dose delay/ reduction or discontinuation of treatment with 
ADCs containing MMAF, DM1, and DM4 $[17,19,20,22,23,27-29,31,32]$. In studies of AGS-16M8F and AGS-16C3F (MMAF-containing ADCs), corneal changes were reversible following treatment cessation, with the time to resolution ranging from a few weeks to several months [23]. The timing of MEC resolution observed in DREAMM-2 and these studies is consistent with the kinetics of stem cell replacement in the cornea in that a minimum of 14 days is required for the epithelial cells to repopulate and normalize the superficial epithelium on the basis of corneal injury (chemical burns) and corneal co-morbidity (penetrating keratoplasty) studies [52, 57-59].

\section{DISCUSSION}

In RRMM, balancing treatment efficacy against the risk of AEs depends on the individual circumstances of each patient. Belamaf is a first-inclass ADC that has demonstrated anti-myeloma activity in patients with RRMM who otherwise have a poor prognosis $[6,7]$. ADCs may represent a paradigm shift in cancer treatment, with four approved agents and more than 200 clinical trials in several types of malignancies [60]. As more patients are treated with ADCs, ophthalmologists are more likely to see these patients for routine examinations and for potential worsening of corneal symptoms. However, ocular AEs are not unique to ADCs; other anti-cancer therapies have a wide range of ocular side effects, including blurred vision, dry eye, conjunctivitis, uveitis, keratitis, keratopathy, ocular pain, peripheral ulcerative keratitis, and serous neuroretinal detachment [61-65]. Although the etiology of ocular side effects associated with anti-cancer therapies may vary, management recommendations generally consist of dose modifications, lubricants, and topical corticosteroids for inflammation [61-63, 65]. Taken together, ocular AEs are not a novel phenomenon in the treatment of cancer, and careful monitoring and management are critical to minimize the occurrence of these events.

MECs, the most frequently observed $\mathrm{AE}$ $(72 \%)$ in patients receiving belamaf in DREAMM-2, were observed in patients with and without symptoms or changes in BCVA and were reversible with dose modification strategies, which is consistent with reports from other ADCs [13]. Corneal findings with belamaf and other ADCs are described as microcyst-like lesions on slit lamp microscopy, though here and in a few other studies IVCM revealed hyperreflective lesions within the corneal epithelium $[15,16]$. These lesions are small, start in the corneal periphery, and, in some patients, migrate toward the center over time and with subsequent infusions. MECs may represent a form of off-target toxicity of belamaf, in which case the involved epithelial cells may be at various stages of apoptosis. In the representative case presented here, the patient also demonstrated whorl-like staining in both eyes, which subsequently resolved. To our knowledge, this has not yet been documented in the context of ADC-MMAF toxicity, though it can be observed with some chemotherapies [66-68].

On the basis of a literature review of MECs with other ADCs and our observations from DREAMM-2, we hypothesize that belamaf enters the cornea, is internalized by basal corneal epithelial cells, and thereafter induces apoptosis of these cells (Fig. 7). Apoptotic cells are extruded, and belamaf-containing epithelial cells that have not yet completed apoptosis migrate centrally and anteriorly. As these cells approach the visual axis, changes in vision may occur. The inhibition of macropinocytosis, a form of nonspecific cellular uptake that has been implicated as a mechanism for off-target AEs of ADCs, appears to be a promising line of inquiry for belamaf but requires further investigation [44].

One limitation of this DREAMM-2 corneal event analysis is the confounding role of corticosteroid eye drops in the resolution of corneal events. The use of prophylactic corticosteroid eye drops was included in the study protocol based on previous studies of other MMAF-containing ADCs, which showed some benefit in managing corneal events [19, 27, 31]. However, the mechanism by which corticosteroid eye drops were expected to mitigate corneal events associated with ADCs is unclear. Further, in the DREAMM-2 ocular sub-study, corticosteroid eye drops were shown to be ineffective in the 
mitigation of MECs [7]. Given that all patients were instructed to use corticosteroid eye drops in at least one eye during belamaf treatment cycles, it is not possible to make definitive conclusions regarding the impact of corticosteroids on the resolution of MECs and symptoms. Another limitation of this analysis is the challenge in obtaining long-term follow-up examination results. For those patients still living, additional follow-up data in this ongoing study is being sought to help confirm the anticipation for near complete resolution of MECs and symptoms over time.

Given we are observing a new corneal condition that has presented as an $\mathrm{AE}$ of a new class of cancer therapies, it is imperative to encourage close collaboration with eye care professionals (including cornea specialists, comprehensive ophthalmologists, and optometrists) and hematologist/oncologists. Eye care professionals will provide the hematologist/oncologists the necessary information needed for he/she to make the appropriate dose modification decisions based on eye examination findings, BCVA assessments, and patientreported symptoms that may impact the patient's vision or quality of life. This will facilitate effective RRMM treatment along with the appropriate management of corneal events [69]. As we continue to learn more about the ocular AEs observed with belamaf and other ADCs, it is important that they are identified and monitored. In "Recommended Monitoring, Diagnosis, and Management Techniques" we outlined our recommended ocular screening and management paradigm based on currently available knowledge.

\section{CONCLUSIONS}

Overall, early data support the favorable antimyeloma activity of belamaf in patients with extensively treated RRMM, who otherwise may have limited or no treatment options [6-9]. Therefore, appropriate identification and management of MECs associated with belamaf are crucial. Here, we provided additional details on the examination features of these MECs and a representative case to help enable the eye care professional to promptly identify these events. We also proposed a mechanism underlying these corneal events observed with ADCs based on a literature review and data from DREAMM-2 that may aid in the investigation of optimal management strategies. Further research is required to determine optimal therapeutic strategies. Proactive management of corneal changes should minimize the burden of these AEs on the patient, enable patients to continue with treatment, and allow better anti-myeloma outcomes in patients treated with belamaf.

Recommended Monitoring, Diagnosis, and Management Techniques

The cumulative incidence of MECs (observed on slit lamp microscopy with or without symptoms or changes in BCVA) in patients receiving the $2.5-\mathrm{mg} / \mathrm{kg}$ dose of belamaf was $72 \%$, with $69 \%$ of patients experiencing MECs by dose 4 and only 2 patients developing a corneal event for the first time after dose 4 . Given the frequency of MECs observed in patients receiving belamaf, it is important to understand its clinical features to better inform the treating hematologist/ oncologist so that he/she is able to make appropriate treatment decisions.

\section{Monitoring}

Conduct eye examinations (visual acuity and slit lamp microscopy) at baseline (up to 3 weeks before), prior to each cycle (up to 2 weeks before), and promptly for worsening symptoms. 


\section{Diagnosis and Staging of MECs}

MECs are very small, hence capturing them by slit lamp microscopic photography takes special consideration. MECs are best observed using retroillumination or indirect illumination at medium to high magnification. In DREAMM-2, patients with MECs in the paracentral or central cornea have been likely to report symptoms (e.g., blurred vision). Table 1 provides guidance for assessing MEC grading based on eye examination findings and changes in BCVA. The eye care professional should provide the treating hematologist/ oncologist with an examination report that includes worst grade for corneal examination finding(s) and decline in BCVA. Determine the recommended dosage modification of belamaf based on the worst finding in the worst affected eye. Worst finding should be based on either a corneal examination finding or a change in visual acuity per the KVA scale.

\section{Management}

The treating hematologist/oncologist should determine the appropriate belamaf dosing based on the highest grade corneal event (based on slit lamp or BCVA assessment) in the most severely affected eye. For grade 1 events, continue treatment at the current dose (Table 1). For grade 2 events, withhold dosing until improvement in corneal examination findings and changes in BCVA to a grade 1 or better event. Resume at the current dose $(2.5 \mathrm{mg} / \mathrm{kg})$. For grade 3 events, withhold treatment until improvement in both corneal examination findings and changes in BCVA to grade 1 or better and resume at a reduced dose. Consider treatment discontinuation for a grade 4 event. Based on a benefit:risk assessment, if continuing treatment with belamaf is being considered, treatment may be resumed at a reduced dose after the event has improved to grade 1 or better event.
Patients with a history of dry eye prior to starting belamaf were more likely to develop moderate/severe MECs compared with patients without a history of dry eye. Advise patients to use preservative-free lubricant eye drops at least 4 times a day starting with the first infusion and continuing until the end of treatment and to avoid use of contact lenses unless directed by an eye care professional. On the basis of available data from the DREAMM-2 study, corticosteroid eye drops are not currently recommended as a prophylactic treatment for MECs. Changes in visual acuity may be associated with difficulty driving and reading. Advise patients to use caution when driving or operating machinery as belamaf may affect their vision.

\section{ACKNOWLEDGEMENTS}

The authors would like to thank the patients who participated in DREAMM-2.

Funding. This study, medical writing support, and the Rapid Service Fee were funded by GlaxoSmithKline. GlaxoSmithKline contributed to study design, implementation, data collection, interpretation, and analysis. Drug linker technology was licensed from Seattle Genetics (Bothell, WA, USA) and the monoclonal antibody was produced with POTELLIGENT Technology licensed from BioWa (Princeton, NJ, USA). Rakesh Popat is supported by the National Institute for Health Research (NIHR) University College London Hospitals (UCLH) Biomedical Research Centre, and this trial was supported in part by the NIHR UCLH Clinical Research Facility and the Cancer Research UK Experimental Cancer Medicine Centre.

Medical Writing, Editorial, and Other Assistance. We thank the ophthalmology and optometry colleagues who provide ophthalmic 
examinations to patients enrolled in the study, and Jeff Jackson, Karrie Wang, Joe Kovach, Kaytlyn Nungesser, Alessandra Tosolini, Eric Lewis, Dave Kleinman, Paul Galette, Juan Pablo Garcia Martinez, and Nazma Ahmed. Medical writing support was provided by Sarah Hauze and Alexandra Staros of Fishawack Indicia, UK.

Authorship. All named authors meet the International Committee of Medical Journal Editors (ICMJE) criteria for authorship for this article, take responsibility for the integrity of the work as a whole, and have given their approval for this version to be published.

Authorship Contributions. Asim V. Farooq, Simona Degli Esposti, Rakesh Popat, Praneetha Thulasi, Sagar Lonial, Ajay K. Nooka, Andrzej Jakubowiak, Douglas Sborov, Brian E. Zaugg, Ashraf Badros, Bennie H. Jeng, Natalie Callander, Kathryn Colby contributed to acquisition of data. Asim V. Farooq, Simona Degli Esposti, Rakesh Popat, Praneetha Thulasi, Sagar Lonial, Brian Zaugg, Bennie H. Jeng, Ira Gupta, Joanna Opalinska, January Baron, Trisha Piontek, Julie Byrne, and Kathryn Colby contributed to data analysis and interpretation. Sagar Lonial, Ira Gupta, Joanna Opalinska, January Baron, and Trisha Piontek contributed to study conception and design.

Disclosures. Asim V. Farooq is a consultant for GlaxoSmithKline; Simona Degli Esposti has received personal fees from GlaxoSmithKline; Rakesh Popat has received grant funding, personal fees, and non-financial support from Takeda; personal fees and non-financial support from Janssen, Celgene, and GlaxoSmithKline, and personal fees from AbbVie; Sagar Lonial has received grant funding and personal fees from Celgene and Takeda, and personal fees from Novartis, Bristol-Myers Squibb, GlaxoSmithKline, Amgen, Merck, and Janssen; Ajay K. Nooka has received grant funding and personal fees from GlaxoSmithKline, Janssen, Bristol-Myers Squibb, Celgene, Takeda, and Amgen; and personal fees from Oncopeptides and Spectrum; Andrzej Jakubowiak consulted for and received honoraria from AbbVie, Adaptive Biotechnologies, Amgen, Bristol-Myers Squibb, Celgene,
Janssen, Juno, and Karyopharm; Douglas Sborov has received personal fees from Celgene, Janssen, and Amgen; Bennie $\mathrm{H}$. Jeng has received compensation from GSK as a consultant; Ira Gupta is an employee of and holds stocks and shares in GlaxoSmithKline and holds stocks and shares in Novartis; Joanna Opalinska, January Baron, and Trisha Piontek are employees of and hold stocks and shares in GlaxoSmithKline; Julie Byrne is an employee of and hold stocks and shares in GlaxoSmithKline and hold stocks and share in Adaptimmune and Novartis; Kathryn Colby is a consultant for GlaxoSmithKline; Praneetha Thulasi, Ashraf Z. Badros, Natalie S. Callander, and Brian E. Zaugg declare no competing interests.

Compliance with Ethics Guidelines. This study was performed in accordance with the Declaration of Helsinki and Good Clinical Practice guidelines following approval by the ethics committees and institutional review boards at each study site: Abramson Cancer Center; MD Anderson Cancer Center, Houston; University of Maryland at Baltimore; Princess Margaret Cancer Centre; Emory University, Winship Cancer Institute; Icahn School of Medicine at Mount Sinai; Universitätsklinikum Tübingen; University of Wisconsin, Carbone Cancer Center; Indiana University Cancer Center; University of Kansas Cancer Center; Memorial Sloan Kettering Cancer Center; Centre Hospitalier Lyon Sud; University of Washington; Huntsman Cancer Institute, University of Utah; Dana-Farber Cancer Institute; Centre Hospitalier Régional Universitaire de Lille Hôpital Claude Huriez; Hôpital Saint-Louis; Centre Hospitalier Universitaire de Bordeaux, Hôpital Haut Lévêque; Universitätsklinikum Würzburg; Clínica Universidad de NavarraPamplona; Levine Cancer Institute, Atrium Health; Centre Hospitalier Universitaire de Nantes - Hôtel Dieu; Azienda OspedalieroUniversitaria di Parma; Ohio State University Comprehensive Cancer Center; University of Melbourne, St Vincent's Hospital; Hospital Quirón Madrid; University College London Hospital, NHS Foundation Trust; Royal Marsen Hospital; University of Chicago; Mayo Clinic, Rochester; Vanderbilt Ingram Cancer Center; 
Oschner Clinic Foundation; University of Calgary; Hospital Universitario de Salamanca; Hospital Morales Meseguer-Murcia; Oxford University Hospitals NHS Trust; Northside Hospital; Queen Elizabeth Hospital; CancerCare Manitoba; Institut Universitaire Du Cancer De Toulouse-Oncopole; Medizinische Hochschule Hannover; Universitätsklinikum Carl Gustav Carus Dresden; Helios Kliniken Schwerin $\mathrm{GmbH}$; Institut für Versorgungsforschung in der Onkologie; Azienda Ospedaliera Universitaria Città della Salute e della Scienza - Presidio Molinette; Centro di Riferimento Oncologico IRCCS; Istituto di Ricovero e Cura a Carattere Scientifico Centro Riferimento Oncologico Basilicata; Institut Català d'Oncologia-Hospital Universitari Germans Trias i Pujol; Hospital Clinic de Barcelona; Hospital Doce de Octubre; Hospital Universitario Doctor Peset; Hospital Virgen de las Nieves; Royal Stoke University Hospital; Nottingham University Hospital Trust - City Campus; Heart of England NHS Foundation Trust; Royal Bournemouth Hospital; Rush University Medical Center; and Smillow Cancer Hospital. All patients provided written informed consent prior to enrollment.

Data Availability. Information about GlaxoSmithKline's data sharing commitments and access requests to anonymized individual participant data and associated documents can be requested for further research from ClinicalStudyDataRequest.com.

Open Access. This article is licensed under a Creative Commons Attribution-NonCommercial 4.0 International License, which permits any non-commercial use, sharing, adaptation, distribution and reproduction in any medium or format, as long as you give appropriate credit to the original author(s) and the source, provide a link to the Creative Commons licence, and indicate if changes were made. The images or other third party material in this article are included in the article's Creative Commons licence, unless indicated otherwise in a credit line to the material. If material is not included in the article's Creative Commons licence and your intended use is not permitted by statutory regulation or exceeds the permitted use, you will need to obtain permission directly from the copyright holder. To view a copy of this licence, visit http://creativecommons.org/licenses/by$\mathrm{nc} / 4.0 /$.

\section{REFERENCES}

1. Palumbo A, Anderson K. Multiple myeloma. N Engl J Med. 2011;364:1046-60.

2. Tai YT, Anderson KC. Targeting B-cell maturation antigen in multiple myeloma. Immunotherapy. 2015;7:1187-99.

3. National Cancer Institute. Surveillance, epidemiology, and end results program. cancer stat facts: myeloma; 2020. https://seer.cancer.gov/statfacts/ html/mulmy.html.

4. Palumbo A, Avet-Loiseau H, Oliva S, et al. Revised international staging system for multiple myeloma: a report from International Myeloma Working Group. J Clin Oncol. 2015;33:2863-9.

5. Mikhael J. Treatment options for triple-class refractory multiple myeloma. Clin Lymphoma Myeloma Leuk. 2020;20:1-7.

6. Gandhi UH, Cornell RF, Lakshman A, et al. Outcomes of patients with multiple myeloma refractory to CD38-targeted monoclonal antibody therapy. Leukemia. 2019;33:2266-75.

7. Lonial S, Lee HC, Badros A, et al. Belantamab mafodotin for relapsed or refractory multiple myeloma (DREAMM-2): a two-arm, randomised, openlabel, phase 2 study. Lancet Oncol. 2020;21:207-21.

8. Trudel S, Lendvai N, Popat R, et al. Targeting B-cell maturation antigen with GSK2857916 antibodydrug conjugate in relapsed or refractory multiple myeloma (BMA117159): a dose escalation and expansion phase 1 trial. Lancet Oncol. 2018;19: 1641-53.

9. Trudel S, Lendvai N, Popat R, et al. Antibody-drug conjugate, GSK2857916, in relapsed/refractory multiple myeloma: an update on safety and efficacy from dose expansion phase I study. Blood Cancer J. 2019;9:37.

10. Tai Y-T, Mayes PA, Acharya C, et al. Novel anti-Bcell maturation antigen antibody-drug conjugate (GSK2857916) selectively induces killing of multiple myeloma. Blood. 2014;123:3128-38.

11. Montes De Oca R, Bhattacharya S, Vitali N, et al. The anti-BCMA antibody-drug conjugate 
GSK2857916 drives immunogenic cell death and immune-mediated anti-tumor responses, and in combination with an OX40 agonist potentiates in vivo activity. HemaSphere. 2019;3:231.

12. Lonial S, Lee HC, Badros A, et al. Pivotal DREAMM2 study: single-agent belantamab mafodotin (GSK2857916) in patients with relapsed/refractory multiple myeloma (RRMM) refractory to proteasome inhibitors (PIs), immunomodulatory agents, and refractory and/or intolerant to anti-CD38 monoclonal antibodies (mAbs). 25th European Haematology Association Annual Congress; June 11-14, 2020; Virtual.

13. Eaton JS, Miller PE, Mannis MJ, Murphy CJ. Ocular adverse events associated with antibody-drug conjugates in human clinical trials. J Ocul Pharmacol Ther. 2015;31:589-604.

14. National Cancer Institute. Common Terminology Criteria for Adverse Events (CTCAE), Version 4.03. Rockwell, MD: National Cancer Institute; 2009. https://ctep.cancer.gov/protocolDevelopment/ electronic_applications/ctc.htm\#ctc_40.

15. Kreps EO, Derveaux T, Denys H. Corneal changes in trastuzumab emtansine treatment. Clin Breast Cancer. 2018;18:e427-e429429.

16. Lee BA, Lee MS, Maltry AC, Hou JH. Clinical and histological characterization of toxic keratopathy from depatuxizumab mafodotin (ABT-414), an antibody-drug conjugate: [RETRACTED]. Cornea. 2018.

17. Younes A, Kim S, Romaguera J, et al. Phase I multidose-escalation study of the anti-CD19 maytansinoid immunoconjugate SAR3419 administered by intravenous infusion every 3 weeks to patients with relapsed/refractory B-cell lymphoma. J Clin Oncol. 2012;30:2776-82.

18. Deklerck E, Denys H, Kreps EO. Corneal features in trastuzumab emtansine treatment: not a rare occurrence. Breast Cancer Res Treat. 2019;175: 525-30.

19. Tannir NM, Forero-Torres A, Ramchandren R, et al Phase I dose-escalation study of SGN-75 in patients with CD70-positive relapsed/refractory non-Hodgkin lymphoma or metastatic renal cell carcinoma. Invest New Drugs. 2014;32:1246-57.

20. Tsuda M, Takano Y, Shigeyasu C, Imoto S, Yamada M. Abnormal corneal lesions induced by trastuzumab emtansine: an antibody-drug conjugate for breast cancer. Cornea. 2016;35:1378-80.

21. Kumar S, Paiva B, Anderson KC, et al. International Myeloma Working Group consensus criteria for response and minimal residual disease assessment in multiple myeloma. Lancet Oncol. 2016;17: e328-e346346.

22. Gan HK, Reardon DA, Lassman AB, et al. Safety, pharmacokinetics, and antitumor response of depatuxizumab mafodotin as monotherapy or in combination with temozolomide in patients with glioblastoma. Neuro Oncol. 2018;20:838-47.

23. Thompson JA, Motzer RJ, Molina AM, et al. Phase I trials of anti-ENPP3 antibody-drug conjugates in advanced refractory renal cell carcinomas. Clin Cancer Res. 2018;24:4399-406.

24. Corbelli E, Miserocchi E, Marchese A, et al. Ocular toxicity of mirvetuximab. Cornea. 2019;38:229-32.

25. Clinical and histological characterization of toxic keratopathy from depatuxizumab mafodotin (ABT414), an antibody-drug conjugate: retraction. Cornea. 2018;37:e55.

26. Donaghy H. Effects of antibody, drug and linker on the preclinical and clinical toxicities of antibodydrug conjugates. MAbs. 2016;8:659-71.

27. Goss GD, Vokes EE, Gordon MS, et al. Efficacy and safety results of depatuxizumab mafodotin (ABT414) in patients with advanced solid tumors likely to overexpress epidermal growth factor receptor. Cancer. 2018;124:2174-83.

28. Lassman AB, van den Bent MJ, Gan HK, et al. Safety and efficacy of depatuxizumab mafodotin + temozolomide in patients with EGFR-amplified, recurrent glioblastoma: results from an international phase I multicenter trial. Neuro Oncol. 2019;21: 106-14.

29. Reardon DA, Lassman AB, van den Bent $M$, et al. Efficacy and safety results of ABT-414 in combination with radiation and temozolomide in newly diagnosed glioblastoma. Neuro Oncol. 2017;19: 965-75.

30. Shapiro GI, Vaishampayan UN, LoRusso P, et al. First-in-human trial of an anti-5T4 antibodymonomethylauristatin conjugate, PF-06263507, in patients with advanced solid tumors. Invest New Drugs. 2017;35:315-23.

31. van den Bent M, Gan HK, Lassman AB, et al. Efficacy of depatuxizumab mafodotin (ABT-414) monotherapy in patients with EGFR-amplified, recurrent glioblastoma: results from a multi-center, international study. Cancer Chemother Pharmacol. 2017;80:1209-17.

32. Banerji U, van Herpen CML, Saura C, et al. Trastuzumab duocarmazine in locally advanced and metastatic solid tumours and HER2-expressing 
breast cancer: a phase 1 dose-escalation and doseexpansion study. Lancet Oncol. 2019;20:1124-35.

33. de Bono JS, Concin N, Hong DS, et al. Tisotumab vedotin in patients with advanced or metastatic solid tumours (InnovaTV 201): a first-in-human, multicentre, phase 1-2 trial. Lancet Oncol. 2019;20: 383-93.

34. Kantarjian HM, Lioure B, Kim SK, et al. A phase II study of coltuximab ravtansine (SAR3419) monotherapy in patients with relapsed or refractory acute lymphoblastic leukemia. Clin Lymphoma Myeloma Leuk. 2016;16:139-45.

35. Martin LP, Konner JA, Moore KN, et al. Characterization of folate receptor alpha (FRalpha) expression in archival tumor and biopsy samples from relapsed epithelial ovarian cancer patients: a phase I expansion study of the FRalpha-targeting antibodydrug conjugate mirvetuximab soravtansine. Gynecol Oncol. 2017;147:402-7.

36. O'Malley DM, Matulonis UA, Birrer MJ, et al. Phase Ib study of mirvetuximab soravtansine, a folate receptor alpha (FRalpha)-targeting antibody-drug conjugate (ADC), in combination with bevacizumab in patients with platinum-resistant ovarian cancer. Gynecol Oncol. 2020;157(2):379-85.

37. Trneny M, Verhoef G, Dyer MJ, et al. A phase II multicenter study of the anti-CD19 antibody drug conjugate coltuximab ravtansine (SAR3419) in patients with relapsed or refractory diffuse large B-cell lymphoma previously treated with rituximab-based immunotherapy. Haematologica. 2018;103:1351-8.

38. Matulonis UA, Birrer MJ, O'Malley DM, et al. Evaluation of prophylactic corticosteroid eye drop use in the management of corneal abnormalities induced by the antibody-drug conjugate mirvetuximab soravtansine. Clin Cancer Res. 2019;25: 1727-36.

39. Beeram M, Krop IE, Burris HA, et al. A phase 1 study of weekly dosing of trastuzumab emtansine ( $\mathrm{T}$ DM1) in patients with advanced human epidermal growth factor 2-positive breast cancer. Cancer. 2012;118:5733-40.

40. Ribrag V, Dupuis J, Tilly H, et al. A dose-escalation study of SAR3419, an anti-CD19 antibody maytansinoid conjugate, administered by intravenous infusion once weekly in patients with relapsed/refractory B-cell non-Hodgkin lymphoma. Clin Cancer Res. 2014;20:213-20.

41. de Goeij BE, Lambert JM. New developments for antibody-drug conjugate-based therapeutic approaches. Curr Opin Immunol. 2016;40:14-23.
42. Mahalingaiah PK, Ciurlionis R, Durbin KR, et al. Potential mechanisms of target-independent uptake and toxicity of antibody-drug conjugates. Pharmacol Ther. 2019;200:110-25.

43. Liu Z, Carvajal M, Carraway CA, Carraway K, Pflugfelder SC. Expression of the receptor tyrosine kinases, epidermal growth factor receptor, ErbB2, and ErbB3, in human ocular surface epithelia. Cornea. 2001;20:81-5.

44. Zhao H, Atkinson J, Gulesserian S, et al. Modulation of macropinocytosis-mediated internalization decreases ocular toxicity of antibody-drug conjugates. Cancer Res. 2018;78:2115-26.

45. Carpenter RO, Evbuomwan MO, Pittaluga S, et al. $\mathrm{B}$-cell maturation antigen is a promising target for adoptive T-cell therapy of multiple myeloma. Clin Cancer Res. 2013;19:2048-60.

46. Hollander DA, Aldave AJ. Drug-induced corneal complications. Curr Opin Ophthalmol. 2004;15: 541-8.

47. Bernitt E, Dobereiner HG, Gov NS, Yochelis A. Fronts and waves of actin polymerization in a bistability-based mechanism of circular dorsal ruffles. Nat Commun. 2017;8:15863.

48. Zhao H, Gulesserian S, Ganesan SK, et al. Inhibition of megakaryocyte differentiation by antibody-drug conjugates (ADCs) is mediated by macropinocytosis: implications for ADC-induced thrombocytopenia. Mol Cancer Ther. 2017;16:1877-86.

49. Peng H, Park JK, Lavker RM. Autophagy and macropinocytosis: keeping an eye on the corneal/ limbal epithelia. Invest Ophthalmol Vis Sci. 2017;58:416-23.

50. Nagai N, Ogata F, Otake H, Nakazawa Y, Kawasaki $\mathrm{N}$. Energy-dependent endocytosis is responsible for drug transcorneal penetration following the instillation of ophthalmic formulations containing indomethacin nanoparticles. Int J Nanomedicine. 2019;14:1213-27.

51. Pennington MR, Saha A, Painter DF, et al. Disparate entry of adenoviruses dictates differential innate immune responses on the ocular surface. Microorganisms. 2019;7:351.

52. Guthoff T, Tietze B, Meinhardt B, Becher J, Guthoff R. Cytosine-arabinoside-induced keratopathy: a model of corneal proliferation kinetics. Ophthalmologica. 2010;224:308-11.

53. Yoon JJ, Ismail S, Sherwin T. Limbal stem cells: central concepts of corneal epithelial homeostasis. World J Stem Cells. 2014;6:391-403. 
54. Lin HP, Singla B, Ghoshal P, et al. Identification of novel macropinocytosis inhibitors using a rational screen of food and drug administration-approved drugs. Br J Pharmacol. 2018;175:3640-55.

55. Fathi AT, Borate U, DeAngelo DJ, et al. A phase 1 study of denintuzumab mafodotin (SGN-CD19A) in adults with relapsed or refractory B-lineage acute leukemia (B-ALL) and highly aggressive lymphoma. Blood. 2015;126:1328.

56. Moskowitz CH, Fanale MA, Shah BD, et al. 182 A phase 1 study of denintuzumab mafodotin (SGNCD19A) in relapsed/refractory B-lineage nonHodgkin lymphoma. Blood. 2015;126:182.

57. Cenedella RJ, Fleschner CR. Kinetics of corneal epithelium turnover in vivo. Studies of lovastatin. Invest Ophthalmol Vis Sci. 1990;31:1957-62.

58. Eghrari AO, Riazuddin SA, Gottsch JD. Overview of the cornea: structure, function, and development. Prog Mol Biol Transl Sci. 2015;134:7-23.

59. Wagoner MD. Chemical injuries of the eye: current concepts in pathophysiology and therapy. Surv Ophthalmol. 1997;41:275-313.

60. Bashraheel SS, Domling A, Goda SK. Update on targeted cancer therapies, single or in combination, and their fine tuning for precision medicine. Biomed Pharmacother. 2020;125:110009.

61. Antoun J, Titah C, Cochereau I. Ocular and orbital side-effects of checkpoint inhibitors: a review article. Curr Opin Oncol. 2016;28:288-94.

62. Heinzerling L, Eigentler TK, Fluck M, Hassel JC, Heller-Schenck D, Leipe J, et al. Tolerability of BRAF/MEK inhibitor combinations: adverse event evaluation and management. ESMO Open. 2019;4: e000491.

63. Huillard O, Bakalian S, Levy C, Desjardins L, Lumbroso-Le Rouic L, Pop S, et al. Ocular adverse events of molecularly targeted agents approved in solid tumours: a systematic review. Eur J Cancer. 2014;50:638-48.

64. Omoti AE, Omoti CE. Ocular toxicity of systemic anticancer chemotherapy. Pharm Pract (Granada). 2006;4:55-9.

65. Srivastava A, Al-Zubidi N, Appelbaum E, Gombos DS, Nader ME, Gidley PW, et al. Immune-Related Oral, Otologic, and Ocular Adverse Events. Adv Exp Med Biol. 2020;1244:295-307.

66. Ding X, Bishop RJ, Herzlich AA, Patel M, Chan CC. Limbal stem cell deficiency arising from systemic chemotherapy with hydroxycarbamide. Cornea. 2009;28:221-3.

67. Kim BY, Riaz KM, Bakhtiari P, et al. Medically reversible limbal stem cell disease: clinical features and management strategies. Ophthalmology. 2014;121:2053-8.

68. Lichtinger A, Pe'er J, Frucht-Pery J, Solomon A. Limbal stem cell deficiency after topical mitomycin C therapy for primary acquired melanosis with atypia. Ophthalmology. 2010;117:431-7.

69. Popat R, Warcel D, O'Nions J, et al. Characterisation of response and corneal events with extended follow-up after belantamab mafodotin (GSK2857916) monotherapy for patients with relapsed multiple myeloma: a case series from the first-time-in-human clinical trial. Haematologica. 2020. https:// doi.org/10.3324/haematol.2019.235937. 